\title{
Ultradeep diamond genesis at Redox conditions of slab-mantle boundary
}

\author{
Jing Gao ${ }^{1,2}$, Bin $\mathrm{Chen}^{1}$, Xiang $\mathrm{Wu}^{3}$ \\ ${ }^{1}$ Hawaii Institute of Geophysics and Planetology, University of Hawaii at Manoa, Honolulu, HI \\ 96822, USA \\ ${ }^{2}$ Key Laboratory of Orogenic Belts and Crustal Evolution, MOE, School of Earth and Space \\ Sciences, Peking University, Beijing 100871, China \\ ${ }^{3}$ State Key Laboratory of Geological Processes and Mineral Resources, China University of \\ Geosciences (Wuhan), Wuhan 430074, China
}

Diamond genesis is an intriguing issue for diamond resources and Earth Carbon Cycle. Besides kimberlitic diamonds, many characteristic ultradeep diamonds hosting inclusions with phase assemblages with a sublithospheric origin have been exploited throughout the world. Ultradeep diamonds with their chemical and inclusion compositions not only record a history of oceanic lithosphere subduction and upward transport at a depth of $>250 \mathrm{~km}$ to $660 \mathrm{~km}$ or deeper, but also indicate their genesis pertinent to mantle-carbonate melts in a $\mathrm{Fe}^{0}$-bufferred reduced condition. In our pilot experiment, the formation of diamonds from $\mathrm{MgCO}_{3}-\mathrm{Fe}^{0}$ system was evidenced in a diamond anvil cell device at $\sim 25 \mathrm{GPa}$ and $\sim 1800 \mathrm{~K}$, the conditions of the depth of $\sim 660$ $\mathrm{km}$ in the mantle. A detailed study of redox mechanism of $\mathrm{MgCO}_{3}-\mathrm{Fe}^{0}$ coupling has been conducted using large volume press along the oceanic lithosphere subduction paths in the pressure-temperature range of 6-25 GPa and 1200-2000 K, covering the formation region of most ultradeep diamonds. The clear reaction boundaries around $\mathrm{Fe}^{0}$-foil in contact with $\mathrm{MgCO}_{3}$ strongly support the redox reaction between carbonatitic slab and $\mathrm{Fe}^{0}$-bearing metals under mantle conditions. Our study experimentally documents the possibility of ultradeep diamond genesis at Redox conditions of carbonateitic slab and $\mathrm{Fe}^{0}$-bearing metals. Furthermore, we will discuss the rates of diamond formation as a function of pressure-temperature conditions. 